\section{European Neurology}

Acute ascending necrotizing myelitis 163

Akinetic rigid syndrome 32

Alzheimer's disease 16, 229

Anti-GM1 103

Apolipoprotein E 229, 234

- - genotype 111

Apraxia, eyelid opening 204

Atrial fibrillation 238

- natriuretic hormone 238

Azathioprine 178

Barbiturate coma 223

Biochemical markers 111

Botulinum toxin 204

Brain 129

- injury 193

Brainstem infarct 200

Cardiac arrhythmias 238

Central tegmental tract 200

Cerebrovascular disease 168

- disorder 174

[123I]ß-CIT SPECT 44

Clinical presentation 182

Cognitive flexibility 193

Complement activation 103

Computed tomography 80

Concentric laminated body 141

Congestive heart failure 238

Creatine kinase activity 154

Cytoplasmic body 141

Deletion 148

Dementia 16, 90, 111, 229

-, Alzheimer type 234

Differential diagnosis 16

Down's syndrome 234

Drug kinetics 223

- monitoring 223

Dual release 119

Dura mater 49

Dystonia 32

Early diagnosis 111

Electroencephalogram 223

ELISA 103

Emotional processing 193

Empathy 193

Environmental sound discrimination 135

Epilepsy surgery 72

Exercise 65

Extrapyramidal disorders 204

Supplement issue No. 1 has its own Subject Index

Filamentous body 141

Focal atrophy 135

Food effect 119

Frontal lobe 193

- - pathology 90

Gene expression 129

Guidelines 3

Guillain-Barré syndrome 141

Hemiageusia 200

Herpes simplex myelitis 163

- zoster oticus 26

Hippocampus 16

Holter ECG system 238

Hypertrophy 49

Ictal symptoms 72

Immunoglobulin 178

Intracranial pressure 49

Intravenous immunoglobulin 3

Isokinetic contraction 218

Lactate 65

Lateralizing value 72

Left superior temporal gyrus 135

Leukoaraiosis 80

Levodopa 119

Linear measurements 16

Lobar atrophy 90

Long-term potentiation 129

Magnetic resonance imaging 9, 16, 26, 38, $49,65,80,163,174$

- - spectroscopy 65

Medial medullary infarction 174

Medulla oblongata 174

MELAS 9

Meningitis 49

Mitochondria 148

Mitochondrial disease 9

- encephalomyopathies 65 mtDNA 148

Multiple sclerosis 178, 182

Muscle biopsy 141

- weakness 218

Myotonia atrophica 238

Necrotic fiber 141

Neurofibrillary tangle 97

Neurological indications 3

- signs 38

Neuropsychology 193
Neuroticism 168

Neurotransmitters 129

Olivary hypertrophy 97

Paraclinical tests 182

Parahippocampal gyrus 16

Parkinson's disease 44, 119, 218

Penicillamine 32

PEO 148

Phagocytosis 141

Pharmacokinetics 119

Phenylalanine 38

Phenylketonuria 38

Phosphorylated neurofilament 97

Polymerase chain reaction 163

Positron emission tomography 9,65

Premotor physiopathology 90

Presynaptic dopaminergic degeneration 44

Primary progressive aphasia 135

Progression 154

Progressive supranuclear palsy 97

Proximal spinal muscular atrophy 154

Pure sensory stroke 211

- word deafness 135

Risk factor 168

Sensation 174

Severity 154

Social behavior 193

Somatosensory evoked potentials 38

Speech 90

Stress 168

Tablet breaking 119

Temporal lobe epilepsy 72

Tenseness 168

Thalamus 211

Therapy 178

Thiopental 223

Topography 174

Transcription factor 129

Traumatic brain injury 223

Treatment 193

Type A behavior 168

Visual evoked potentials 38

- rating scales 80

White matter changes 80

Wilson's disease 32

\section{KARGER}

Fax +4161306 1234

E-Mail karger@karger.ch www.karger.com
(C) 1998 S. Karger AG, Basel

This article is also accessible online at: http://BioMedNet.com/karger 